\title{
Evidence-based prescribing for post-traumatic stress disorder
}

\author{
Jonathan I. Bisson, Amy Baker, William Dekker and Mathew D. Hoskins
}

\section{Summary}

There is strong research evidence to support the pharmacological treatment of post-traumatic stress disorder (PTSD) as a second line to trauma-focused psychological interventions. Fluoxetine, paroxetine, sertraline and venlafaxine are the bestevidenced drugs, with lower-level evidence for other medications. It is important that prescribing for PTSD is evidence-based.

\section{Declaration of interest}

None.

\section{Keywords}

PTSD; pharmacotherapy; prescribing algorithm; treatment; evidence-based.

\section{Copyright and usage}

(c) The Author 2020.
Jonathan I. Bisson (pictured) is a practising psychiatrist involved in traumatic stress research and he is chair of the International Society for Traumatic Stress Studies' PTSD Treatment Guidelines Committee. Amy Baker and William Dekker are medical students at Cardiff University, UK. Mathew D. Hoskins is a practising psychiatrist involved in traumatic stress research.

Post-traumatic stress disorder (PTSD) is common and associated with significant distress and impaired functioning. Thankfully, a number of psychological and pharmacological treatments have been shown to help. Trauma-focused psychological interventions in the form of cognitive-behavioural therapies with a trauma focus (CBT-TF) and eye-movement desensitisation and reprocessing (EMDR) are recommended as the treatments of choice for PTSD by most guidelines, ${ }^{1}$ including those recently published by the UK's National Institute for Health and Care Excellence (NICE) ${ }^{2}$ and the International Society for Traumatic Stress Studies (ISTSS). ${ }^{3}$ Pharmacological approaches are recommended as second-line treatments but medication is widely prescribed for PTSD and it is important that it is prescribed in an evidence-based manner.

\section{Are psychological treatments more effective than pharmacological treatments?}

As suggested by guideline recommendations, the magnitude of benefit of pharmacological interventions has been found to be inferior to that of trauma-focused psychological interventions; small mean effect sizes of less than 0.4 are found for the most effective drugs and large mean effect sizes of over 1.2 for CBT-TF and $\mathrm{EMDR}^{2,3}$ It is, however, notoriously difficult to directly compare the results of randomised controlled trials (RCTs) of psychological and pharmacological approaches owing to important methodological differences.

In contrast to the pharmacological placebo, it is very difficult to design and provide a convincing psychological placebo and virtually impossible to mask providers and recipients to what psychological intervention is being given and received. The meta-analyses that underpin PTSD guideline recommendations for psychological treatments use data from RCTs with a waiting-list or usual-care control. The meta-analyses for pharmacological treatments use data from RCTs with a placebo control. Demonstrating efficacy against a placebo is more difficult to achieve than against a waiting-list control and, therefore, the apparent magnitude of superiority of psychological over pharmacological treatments for PTSD is likely to be overestimated by considering raw effect size estimates alone.

The ISTSS prevention and treatment guidelines for PTSD took the likely influence of different control conditions into account by setting a lower effect size threshold for the strong recommendation of treatments evaluated through placebo-controlled RCTs than for those evaluated through RCTs with waiting-list/usual-care controls. ${ }^{3}$ The correct level at which to set such thresholds is, however, unknown and open to debate. The ISTSS effect size thresholds were 0.4 for placebo-controlled trials and 0.8 for those with waiting-list/ usual-care controls; these were set by a committee of experts and therefore need to be interpreted with a degree of caution.

A strong placebo effect has been found in PTSD treatment trials. In one of the two RCTs of venlafaxine that contributed to the ISTSS and NICE guidelines, participants receiving venlafaxine experienced a mean reduction of $64 \%$ in their clinician-rated PTSD symptoms compared with $54 \%$ for those who received placebo. This resulted in a small mean effect size of around 0.3 , clearly underestimating the real benefit that people with PTSD who took venlafaxine experienced, albeit probably accurately representing the proportion of that benefit directly attributable to the unique chemical composition of venlafaxine. How to capture the placebo effect without prescribing a placebo remains a challenging conundrum faced in many areas of healthcare. There is some evidence that the placebo effect is stronger in the acute phase of treatment and tends to wane, with true medication effects lasting longer, so RCTs of longer duration could better tease out the difference.

\section{What do PTSD treatment guidelines recommend?}

The current NICE guidelines ${ }^{2}$ recommend selective serotonin reuptake inhibitors (SSRIs) as a group and venlafaxine as the first-line pharmacological treatments for PTSD. The ISTSS guidelines, ${ }^{3}$ like the original NICE guidelines, considered individual drugs separately and found fluoxetine, paroxetine and sertraline to be the only SSRIs with definite efficacy and therefore recommend these three by name, alongside the serotonin-noradrenaline reuptake inhibitor (SNRI) venlafaxine, as the pharmacological treatments of choice for PTSD.

Both the NICE and ISTSS guidelines recommend antipsychotic medications as second-line pharmacological approaches. NICE 
does not differentiate between antipsychotics; ISTSS recommends quetiapine alone, as it was the only antipsychotic found to have any evidence of efficacy as a monotherapy and this was limited to one RCT with a total of 80 participants.

Ultimately, the differences in recommendations between these and the other major PTSD treatment guidelines are minor and they provide a clear and relatively consistent prescribing message. ${ }^{1}$

\section{Implementing PTSD treatment guideline recommendations in practice}

It seems highly likely that more people with PTSD would benefit from medication if it were prescribed according to the current evidence base. Guidelines should facilitate this but, sadly, rarely provide sufficient detail to allow clinicians to determine the optimal way to prescribe recommended drugs. This probably contributes to their lack of widespread implementation and to sub-optimal prescribing. To address this gap, we developed the Cardiff PTSD Prescribing Algorithm (available in the supplementary material at https://doi.org/10.1192/bjp.2020.40) to help clinicians make appropriate decisions about the pharmacological treatment of people with PTSD, primarily based on the ISTSS evidence-based recommendations. The algorithm is designed for PTSD as opposed to complex PTSD (CPTSD) but, given the absence of evidence with respect to the pharmacological treatment of CPTSD specifically, and the overlap between PTSD and CPTSD, it also represents an evidence-informed approach to the pharmacological treatment of CPTSD.

To determine appropriate guidance for the algorithm, we considered the dosing regimens used in all the RCTs included in the meta-analyses that led to the ISTSS and NICE recommendations. Interestingly, the mean doses of all the recommended drugs used approached the maximum dose determined in the British National Formulary. ${ }^{4}$ This suggests that a significant number of people with PTSD will only benefit optimally from recommended pharmacological treatments if they take higher doses of them.

As part of the development of the ISTSS guidelines, a systematic review of augmentation of pharmacological therapy with other pharmacological agents was undertaken. This revealed evidence that prazosin, an alpha- 1 adrenoceptor antagonist, and risperidone can improve PTSD symptoms when added to another medication (usually an SSRI or SNRI). This allowed us to include evidencebased guidance on what to do if a person with PTSD does not fully respond to monotherapy. We were also able to include drugs with lower levels of RCT evidence (e.g. from one small RCT alone) as possible later pharmacological options and provide guidance on the management of specific problematic symptoms that often present with PTSD but may need addressing separately (e.g. marked agitation and insomnia).

The algorithm encourages a measurement-based approach to care, not least given the evidence that this is a more reliable way to prescribe antidepressants than relying on clinician judgement alone. ${ }^{5}$ After assessment and a fully informed decision by the person with PTSD to receive medication, it is recommended that fluoxetine, paroxetine or sertraline is tried, with escalation of dose at monthly intervals depending on response and tolerability. Venlafaxine is advocated as second line, with either prazosin or quetiapine (preferred to risperidone on account of side-effect profiles) augmentation the third line. If a person with PTSD either does not want or is unable to tolerate an antidepressant, then it would be reasonable to use prazosin or quetiapine as monotherapy. The final step is to consider drugs from other classes with less evidence of effect (e.g. amitriptyline or phenelzine). In addition to these suggested steps, the algorithm provides information on side-effect profiles, monitoring requirements and specific issues concerning the initiation and dose escalation of prazosin.

\section{Conclusions}

At present, four medications (fluoxetine, paroxetine, sertraline and venlafaxine) have robust evidence of low effect for people with PTSD, making them an important treatment option and one that clinicians who see people with PTSD should be familiar with. A number of other evidence-based pharmacological options also exist. Despite trauma-focused psychological interventions being the first-line treatment, most people with PTSD who present for help will be prescribed psychotropic medication and there are a number of reasons why pharmacological treatment should be considered. These include: evidence of effect; personal choice; waiting lists for psychological treatment; factors, such as lack of stability, that prevent psychological treatment; presence of comorbidity/specific symptom profiles; and ongoing symptoms despite psychological treatment.

More work is needed to develop more effective pharmacological treatments for PTSD but a systematic and measurement-based approach to prescribing, based on the current evidence, would be likely to reduce the burden of PTSD and lead to improved health and well-being for people with this condition.

Jonathan I. Bisson (D, DM, FRCPsych, Professor of Psychiatry, School of Medicine, Cardiff University; Amy Baker, Medical Student, School of Medicine, Cardiff University; William Dekker, BSC, Medical Student, School of Medicine, Cardiff University; Mathew D. Hoskins, MBBCh, Consultant Psychiatrist, Cardiff and Vale University Health Board, Cardiff, UK.

Correspondence: Professor Jonathan I. Bisson. Email: bissonji@cf.ac.uk

First received 19 Nov 2019, final revision 17 Jan 2020, accepted 17 Jan 2020

\section{Supplementary material}

Supplementary material is available online at https://doi.org/10.1192/bjp.2020.40

\section{References}

1 Hamblen JL, Norman SB, Sonis J, Phelps A, Bisson J, Nunes V, et al. A guide to guidelines for the treatment of posttraumatic stress disorder in adults: an update. Psychotherapy 2019; 56: 359-73.

2 National Institute for Health and Care Excellence. Post-Traumatic Stress Disorder (NICE Guideline NG116). NICE, 2018.

3 Bisson Jl, Berliner L, Cloitre M, Forbes D, Jensen TK, Lewis C, et al. The International Society for Traumatic Stress Studies new guidelines for the prevention and treatment of PTSD: methodology and development process. J Trauma Stress 2019; 32: 475-83.

4 Joint Formulary Committee. BNF: British National Formulary No. 78: September 2019-March 2020. Royal Pharmaceutical Society, 2019.

5 Guo T, Xiang Y-T, Xiao L, Hu C-Q, Chiu HFK, Ungyari GS, et al. Measurementbased care versus standard care for major depression: a randomized controlled trial with blind raters. Am J Psychiatry 2015; 172: 1004-13. 